\section{Sur l'unité d'origine du bleu de l'eau.}

(Bulletins de l'Académie royale de Belgique [Classe des sciences], no 2, pp. 72-80, 1899.)

On le sait, plusieurs physiciens ont regardé la couleur des mers et de certains lacs comme n'appartenant pas en propre à l'eau, mais comme étant produite par la réflexion de la lumière du jour sur des particules invisibles que l'eau tient toujours en suspension. Le point de départ de cette manière de voir se trouvait dans la théorie alors admise sur l'origine du bleu du ciel. L'atmosphère passant pour incolore, il avait été nécessaire de chercher, en dehors des phénomènes d'absorption caractéristiques des substances colorées, une cause à la coloration du firmament. On a cru trouver celle-ci dans une réflexion sélective des ondes bleues de la lumière blanche sur des particules extrêmement petites dont on admettait l'existence dans l'air.

J'ai montré, dans un travail récent (*), que cette théorie, tout irréprochable qu'elle soit au point de vue mathématique, ne se vérifie cependant pas par l'observation.

Le problème de la couleur des eaux a eu une genèse semblable. L'observation journalière des masses d'eau peu considérables nécessaires à nos besoins nous a aussi d'abord fait regarler ce liquide comme le type d'une substance incolore; on a été naturellement porté à attribuer la coloration des mers et des lacs à des phénomènes d'interférence ou de réflexion spéciale. Bien que $H$. Davy et, après lui, Bunsen eussent fait voir que l'eau pure n'est pas absolument incolore, divers savants, notammant Hagenbach, Tyndall, Soret, ont

(*) Bulletin de l'Académie royale de Belgique, 3e série, t. XX̊XVI, p. 504, 1898. regardé la couleur des eaux comme ayant, en tout ou en partie, une origine semblable au bleu du ciel; elle devait ètre le résultat de la réflexion de la lumière du jour sur des particules suspendues dans l'eau, de dimensions trop petites pour renvoyer les rayons de grande longueur d'onde. Comme preuve à l'appui, on a signalé le fait que la lumière renvoyée par les couches de dessous la surface des eaux était polarisée comme la lumière du ciel. On oubliait cependant que le phẻnomène de la polarisation ne prouvait pas nécessairement que la lumière réfléchie fût bleue.

Devant la divergence des idées qui régnaient alors sur l'origine de la couleur des eaux, j'ai désiré me renseigner par des expériences nouvelles sur la valeur de l'une et de l'autre théorie. Mes observations firent voir $\left(^{*}\right)$ que l'eau est véritablement bleue par elle-même et que les fines particules qu'elle tient en suspension, tout en contribuant principalement à son illumination, n'ont cependant qu'une influence inappréciable, sinon nulle, sur l'intensité du bleu; toutefois ces particules peuvent être la cause des tons verts plus ou moins prononcés, observés dans certaines eaux de la nature, parce que la lumière qui traverse un milieu trouble devient plus ou moins jaunebrun. Celte coloration associée au bleu de l'eau donne le vert.

Mes recherches avaient ainsi confirmé une opinion déjà émise par L. Soret $\left(^{* *}\right)$ en $\mathbf{1 8 6 9}$, mais qui m'avait échappé. Elle se trouvait en note au bas de la page $\mathbf{1 1 6 9}$ du travail de ce savant, sur l'Illumination des corps transparents; je me fais un devoir de la reproduire : «Quant à la couleur de l'eau..., dit Soret, je suis arrivé à croire que ces particules en suspension n'ont qu'une influence secondaire : elles modifient bien d'une manière importante l'apparence et la teinte de. l'eau, mais on ne peut leur attribuer l'origine même de la coloration bleue. ')

Pourtant, ni les travaux de Soret ni les miens ne paraissent avoir éclairé suffisamment le rôle des particules en suspension pour rallier l'opinion générale. Dans un article récent sur la couleur des mers et des.lacs $\left.{ }^{(* *}\right)$, M. le professeur $\mathrm{Abegg}$ dit explicitement que ceux-ci

(*) Bulletin de l'Académie royale de Belgique, 3e série, t. V, pp. 5̌-84, 1883, et t. XII, pp. 814-857, 1886 .

$\left.{ }^{* *}\right)$ Comptes rendus, t. LXIX, pp. 1192-1196, 1869.

$\left.{ }^{* * *}\right)$ Naturwissenschaftl. Rundschau, t. XIII, p. 169, 1898. 
sont bleus par suite du concours de deux causes différentes : d'abord parce que l'eau est bleue et ensuite parce que la réflexion de la lumière sur les particules suspendues doit produire le bleu. M. Abegg ne fournit pas de preuves nouvelles à l'appui de son affirmation; il se réfère aux travaux de Tyndall et de lord Rayleigh sur' l'origine du bleu du ciel, en ajoutant que si l'on reporte la théorie des savants anglais à l'eau, il s'ensuit nécessairement que, même sans la couleur propre de ce liquide, l'eau limpide devrait être bleue. Cette déduction serait même démontrée physiquement, selon lui, parce que L. Soret et Hagenbach ont constaté la polarisation de la lumière de l'eau de certains lacs. A mon avis, M. Abegg va trop loin dans sa conclusion. Les observations des physiciens suisses prouvent incontestablement que la lumière émanant de l'eau est de la lumière réfléchie, mais en aucune façon que la réflexion ait pour conséquence l'extinction de la partie complémentaire du bleu de l'eau. Comme je l'ai fait d'ailleurs moi-même dans mon premier article sur ce sujet, M. Abegg met trop d'absolu dans l'opinion qu'il attribue à Soret. L'éminent physicien de Genève n'a pas prétendu, nous l'avons vu plus haut, que la réflexion de la lumière sur les particules suspendues dans l'eau produisît un effet d'importance semblable à la coloration due à l'absorption. Soret s'est, au surplus, expliqué à nouveau sur ce point en $1884\left(^{*}\right)$.

Quoi qu'il en soit, M. Abegg' regarde la théorie de la réflexion comme aussi certaine et aussi irréfutable que la théorie de l'absorption (ebenso sicher richtig und unwiederleglich), et il admet que la couleur bleue de l'eau doit nécessairement avoir une double origine. On le voit, M. Abegg replace la théorie de la couleur des eaux au point où elle était avant mes premières expérienceś en ce qui concerne le bleu $\left({ }^{* *}\right)$. Il est donc bien naturel que j’aie tenu à vérifier si

(*) Archives des sciences physiques et naturelles (3), t. XI, pp. 276-296, 1884.

(**) Le Profr Abegg explique l'apparition des tons verts de certaines eaux par ce fait que la lumière pénétrerait moins profondément dans les eaux vertes, plus troubles, et qu'elle ne pourrait pas perdre alors, par absorption, une proportion suftisante de ses rayons à grande longueur d'onde. Cette explication ne se vérifie pas par l'expérience. J'ai montré, devant le Ve Congrès international d'hydrologie, de géologie et de climatologic, tenu à liége en septembre dernier, que la teinte bleue de l'eau ne vire jamais au vert quand on diminue la longueur de la course de la lumière. Je reviendrai du reste sur ce point dans un article spécial. mes observations étaient véritablement sans portée et si la réflexion de la lumière intervenait d'une manière certaine et irréfutable dans la production de la couleur bleue.

Ce sont les expériences que j’ai instituées à ce sujet que je désire faire connaitre à présent. On verra qu'elles confirment mes conclusions antérieures en établissant l'unité d'origine du bleu de l'eau.

\section{$*^{*} *$}

Le but à atteindre dans ces expériences était la connaissance pratique, et non théorique, des propriétés optiques des particules qui forment le trouble invisible des eaux limpides.

Je reconnais volontiers avec les mathématiciens que des particules dont les dimensions sont de l'ordre de grandeur des ondes lumineuses, pourront mieux réfléchir les ondes courtes; mais il n'est pas démontré que les particules suspendues dans les eaux remplissent nécessairement les conditions supposées. Si Tyndall a vu de la lumière bleue en éclairant un mélange de vapeurs de certains éthers nitreux et d'acide chlorhydrique, il n'est pas certain que ce bleu fût dû à une simple réflexion, car il n'a duré que le temps de la réaction. chimique provoquée par la lumiere. Et les conclusions de Tyndall fussent-elles même exactes, l'assimilation du trouble des eaux à un nuage naissant resterait gratuite aussi longtemps qu'une vérification ne serait pas intervenue.

J'ai donc monté une lanterne de Duboscq, à éclairage électrique puissant, pour produire l'irradiation du trouble de l'eau d'après le procédé de Tyndall et le rendre visible de cette façon. La lampe était alimentée par un courant de 12 ampères et 110 volts, et placée au foyer des lentilles de la lanterne, de manière à produire autant que possible un faisceau de rayons lumineux parallèles.

Au-devant de l'objectif, j'ai disposé un tube en verre de $\mathbf{1}^{\mathrm{m}} 20$ de long et $0^{\mathrm{m}} 07$ de diamètre intérieur, fermé par des plans minces de cristal.

Ce tube a été rempli d'eau distillée parfaitement limpide à la lumière du jour, ou bien, pour varier, d'eau alimentaire de la ville de Liége, également limpide.

Jamais ces eaux ne se sont montrées optiquement vides : dans chacune, le faisceau lumineux était visible dans toute la longueur du 


$$
-818-
$$

tube et, chose curieuse, l'eau distillée du laboratoire rendait le faisceau plus visible que l'eau alimentaire. Elle donnait, pour ainsi dire, une traînée estompée continue, tandis que la dernière en montrait une moins opaque, moins nourrie et partant malaisée à découvrir. L'eau distillée est donc moins propre que l'eau alimentaire $\left(^{*}\right)$.

J'ai rempli aussi le tube d'ean de pluie, clarifiée par le repos, en vue d'opérer sur un liquide ayant pu se charger plus abondamment encore des poussières de l'air que l'eau distillée. Le faisceau lumineux s'est montré, comme je le présumais, avec plus d'évidence que dans l'eau distillée.

Dans chacun de ces liquides, la trânée lumineuse était blanc laiteux plus ou moins visible, mais elle n'a fait voir aucun ton rappelant le bleu de l'eau ou le bleu du ciel. Tyndall disait à propos de l'illumination de ses nuages naissants que son tube " reproduisait un coin du ciel »; ici, rien ne rappelait l'azur de l'eau, même dans sa plus grande atténuation.

Ce point étant acquis par des observations répétées, j’ai interposé entre le tube et la source lumineuse une auge chargée d'une solution de fuchsine, concentrée au point de ne laisser passer que la région rouge de la partie visible du spectre solaire. Le liquide se trouvait done éclairé exclusivement par de la lumière rouge. Dans ces conditions, la trainée lumineuse a apparu rouge, sans que sa visibilité en fût atteinte en rien.

Étant donné que les substances fluorescentes transforment les ondes lumineuses incidentes en ondes plus longues et non plus courtes, cette expérience prouve que la trainée n'était pas due à la fluorescence, sinon elle aurait dû devenir invisible ou prendre un ton èn rapport avec les rayons très réfrangibles que laisse encore passer la fuchsine. Cette conclusion se base sur ce qu'une solution de sulfate de quinine, convenablement étendue, a persisté dáns sa fluorescence bleue dans la lumière élecirique, malgré l'interposition de la fuchsine.

Dans d'autres expériences, j'ai placé sur le passage de la lumière incidente une solution alcoolique jaune d'acide picrique, qui éteignait complètement l'extrémité violette du spectre et ne laissait passer que

$(*)$ Je montrerai à quelle cireonstance ce fait est dû́, dans une note sứ la préparation des liquides optiquement vides, qui fera suite à cet article. le rouge, l'orange, le jame et le vert, avec une frange insignifiante de bleu. La trainée lumineuse des eaux s'est montrée aussi intense, mais elle était jaune. Soit dit comme contrôle, cette lumière jaune de l'acide picrique éteignait absolument la fluorescence du sulfate de quinine.

Enfin, j'ai éclairé les eaux avec une lumière verte obtenue par interposition d'une solution concentrée de chlorure de nickel, puis avec une lumière bleue d'un verre coloré au cobalt ou d'une solution d'hydrate cuivrique dans l'ammoniaque. Le résultat a été constamment le même : dans la lumière verte, le faisceau lumineux était vert, et dans la lumière bleue, il est devenu bleu, sans variation d'intensité.

$$
*^{*} *
$$

Il résulte à toute évidence de ces observations, me paraît-il, que les particules auxquelles l'eau limpide, distillée ou naturelle, doit son illumination, ont la propriété de réfléchir, a vec une égale facilité, les ondes rouges, jaunes, vertes, bleuès, et qu'elles ne peuvent dès lors causer la coloration bleue de l'eau. Elles nous renvoient la lumière du jour sans altération chromatique. Les spéculations des mathématiciens sur la génération de la lumière bleue ne se vérifient donc pas plus par l'expérience en ce qui concerne l'eau qu'elles ne se vérifient pour l'origine du bleu du ciel. Il est entendu toutefois que les observations présentes n'infirment en rien les calculs de physique mathématique; mais elles prouvent que les conditions matérielles admises par les théoriciens ne sont pas réalisées dans la nature et que, par suite, les déductions tirées de ces conditions doivent demeurer sans emploi pratique.

En résumé, il m'est permis de regarder mes recherches antérieures comme ayant reçu une nouvelle confirmation : l'eau est bleue par elle-méme et les particules qu'elle tient en suspension causent principalement son illumination; selon leur nature, elles déterminent aussi les modifications de la couleur de fond et produisent les tons verdâtres lorsqu'elles n'ont pas pour effet de supprimer toute coloration visible, ainsi que je l'ài montré récemment. 